\title{
Differential Regulation of Dopaminergic Gene Expression by $\operatorname{Er} 81$
}

\author{
John W. Cave, ${ }^{1,2}$ Yosuke Akiba, ${ }^{1}$ Kasturi Banerjee, ${ }^{1}$ Shivraj Bhosle, ${ }^{1}$ RoseAnn Berlin, ${ }^{1}$ and Harriet Baker ${ }^{1,2}$ \\ ${ }^{1}$ Burke Medical Research Institute and ${ }^{2}$ Department of Neurology and Neuroscience, Weill Cornell Medical College, White Plains, New York 10605
}

A recent study proposed that differentiation of dopaminergic neurons requires a conserved "dopamine motif" (DA-motif) that functions as a binding site for ETS DNA binding domain transcription factors. In the mammalian olfactory bulb (OB), the expression of a set of five genes [including tyrosine hydroxylase $(T h)$ ] that are necessary for differentiation of dopaminergic neurons was suggested to be regulated by the ETS-domain transcription factor ER81 via the DA-motif. To investigate this putative regulatory role of ER81, expression levels of these five genes were compared in both olfactory bulbs of adult wild-type mice subjected to unilateral naris closure and the olfactory bulbs of neonatal Er81 wild-type and mutant mice. These studies found that ER81 was necessary only for Th expression and not the other cassette genes. Chromatin immunoprecipitation (ChIP) and electrophoretic mobility shift assays (EMSA) experiments showed that ER81 bound directly to a consensus binding site/DA-motif in the rodent Th proximal promoter. However, the ER81 binding site/DA-motif in the Th proximal promoter is poorly conserved in other mammals. Both ChIP assays with canine OB tissue and EMSA experiments with the human Th proximal promoter did not detect ER81 binding to the Th DA-motif from these species. These results suggest that regulation of Thexpression by the direct binding of ER81 to the Th promoter is a species-specific mechanism. These findings indicate that ER81 is not necessary for expression of the $\mathrm{OB}$ dopaminergic gene cassette and that the DA-motif is not involved in differentiation of the mammalian OB dopaminergic phenotype.

\section{Introduction}

Dopaminergic neurons express a minimal set of five genes that are collectively necessary for the biosynthesis of dopamine [tyrosine hydroxylase (Th), GTP cyclohydrolase (Gtpch), and aromatic amino acid decarboxylase (Aadc)], as well as its uptake and storage [dopamine transporter (Dat) and vesicular monoamine transporter 2 (Vmat2)]. However, expression levels for each of these genes differs between the various groups of dopaminergic neurons in the mammalian brain (Weihe et al., 2006). Also, the dopaminergic groups differ in their spatial and temporal developmental origins, their molecular and morphological features, as well as the neurological functions they mediate (Cave and Baker, 2008). These differences between dopamine cell groups raises the question of whether a common molecular mechanism regulates expression of this minimal set of five dopaminergic genes (or gene cassette).

Recent studies found that the ETS DNA binding domain transcription factor Ast-1 was necessary for expression of the dopaminergic gene cassette in Caenorhabditis elegans (Flames and Hobert, 2009). These studies also identified a putative ETS DNA binding site, the "dopamine motif" (DA-motif), near the transcription start site of each gene in the cassette. Although direct binding of Ast-1 to the DA-motif was not shown, the identifica-

\footnotetext{
Received Jan. 25, 2010; accepted Feb. 19, 2010.

This work was supported by National Institutes of Health Grant R01DC008955 and the Burke Medical Research Institute.

Correspondence should be addressed to Harriet Baker, Department of Neurology and Neuroscience, Weill Cornell Medical College, Burke Medical Research Institute, White Plains, NY 10605. E-mail: habaker@med.cornell.edu. DOI:10.1523/JNEUROSCI.0419-10.2010

Copyright $\odot 2010$ the authors $\quad 0270-6474 / 10 / 304717-08 \$ 15.00 / 0$
}

tion of DA-motifs near mammalian dopamine cassette genes suggested that ETS-domain protein/DA-motif regulatory logic is an evolutionarily conserved mechanism for mediating dopaminergic neuronal differentiation.

Dopaminergic interneurons in the mammalian olfactory bulb (OB) are located almost exclusively in the glomerular layer. They are distinct from other dopaminergic neurons in the mammalian brain because they are generated throughout life from neural stem cells in the subventricular zone (SVZ). Although the OB dopaminergic interneurons are initially derived from the embryonic lateral ganglionic eminence (LGE) (Anderson et al., 1999; Wichterle et al., 2001; Stenman et al., 2003), the vast majority of these neurons are generated in the postnatal SVZ (Luskin, 1993; Lois and Alvarez-Buylla, 1994), with a peak at $\sim 5 \mathrm{~d}$ after birth (Hinds, 1968).

Er81 (Etv1) is coexpressed with Th in the mouse OB (Allen et al., 2007; Saino-Saito et al., 2007) and has been proposed to be part of a "molecular code" that specifies OB dopaminergic interneurons (Allen et al., 2007). Because Er81 is an ortholog of C. elegans ast-1, ER81 has been suggested to regulate differentiation of mammalian OB dopaminergic interneurons via the DA-motif (Flames and Hobert, 2009). Consistent with this possibility, the DA-motif sequence overlaps with the previously established ER81 consensus binding site (Brown and McKnight, 1992).

In the present study, the necessity of Er81 for dopaminergic gene cassette expression in the $\mathrm{OB}$ was examined in both neonatal Er81 mutant mice and adult wild-type mice subjected to a unilateral naris closure. Phylogenetic analyses of several mammalian Th proximal promoter sequences as well as both chromatin immunoprecipitation (ChIP) and electrophoretic mobility shift 

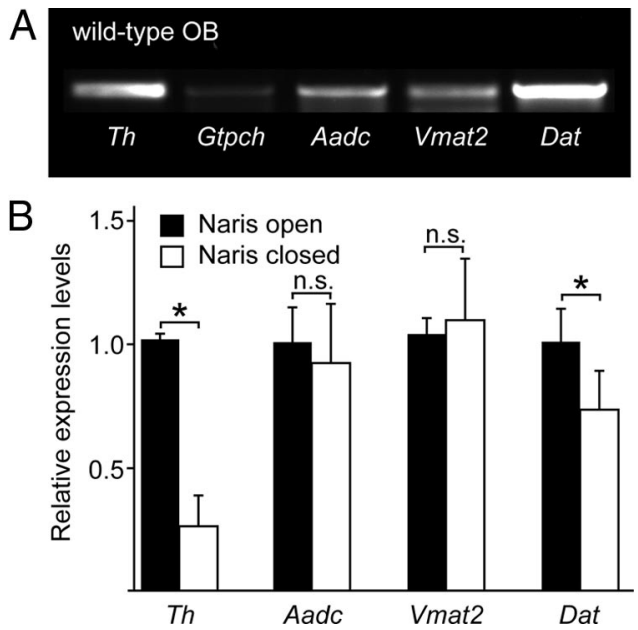

Figure 1. Expression of the dopaminergic gene cassette members in a unilateral naris closed mouse model of odor deprivation. $A$, Qualitative analysis of dopamine gene cassette expression levels in the $\mathrm{OB}$ of an adult wild-type mouse. The Th and Dat genes are expressed at the highest levels. Aadc and Vmat2 are substantially lower, and Gtpch is nearly undetectable. $\boldsymbol{B}$, Relative expression levels of dopamine gene cassette members in the $\mathrm{OB}$ either ipsilateral or contralateral to naris closure as measured by quantitative RT-PCR. For each gene, expression levels ipsilateral to naris closure are shown relative to the levels in the contralateral OB. Gtpch expression was too low for reliable measurement and was not included in the analysis. Expression levels for both $T h$ and Dat were significantly decreased in the $O B$ ipsilateral to naris closure (indicated by asterisk), whereas levels for Aadc and Vmat2 did not significantly differ (n.s.).

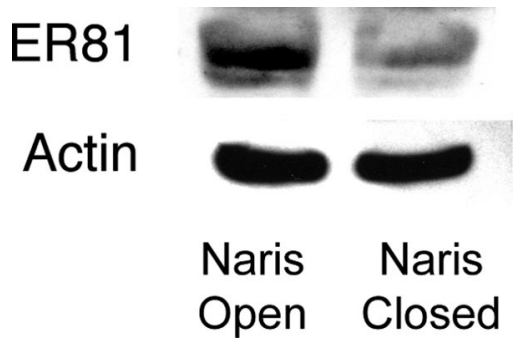

Figure2. ER81 expression in a unilateral naris closed mouse model of odor deprivation. ER81 protein levels were substantially decreased in the $0 B$ ipsilateral to naris closure.

assays (EMSAs) were performed to establish whether ER81 regulated Th expression in the OB by directly binding to a DA-motif/ ER81 consensus binding site in the Th proximal promoter.

\section{Materials and Methods}

Animals. Er81 mutant mice were obtained from Dr. Thomas Jessell (Columbia University, New York, NY) (Arber et al., 2000). Mice were housed in humidity-controlled cages at $22^{\circ} \mathrm{C}$ under a $12 \mathrm{~h}$ light/dark cycle and provided with food and water ad libitum. OB tissue from dogs was kindly provided by Dr. Paul Heerdt (Weill Cornell Medical College, New York, $\mathrm{NY}$ ). All procedures were performed under protocols approved by the Cornell University Institutional Animal Care and Use Committee and conformed with National Institutes of Health guidelines.

Unilateral naris closure mouse model of odor deprivation. One nostril of wild-type C57BL/6J mice (aged 6-8 weeks) was surgically closed using a spark-gap cautery under pentobarbital anesthesia. Naris occlusion was confirmed at 1 and 3 months after operation. Details of the naris occlusion procedure have been published previously (Baker et al., 1993; Liu et al., 1999).

Reverse transcriptase-PCR. RNA was isolated from mouse OBs using an RNA mini-prep kit (Qiagen) following the protocol of the manufacturer. For nonquantitative analysis, RNA was collected from two adult wild-type C57BL/6J mice. For quantitative analysis of dopamine gene cassette expression levels, RNA was isolated from either four adult

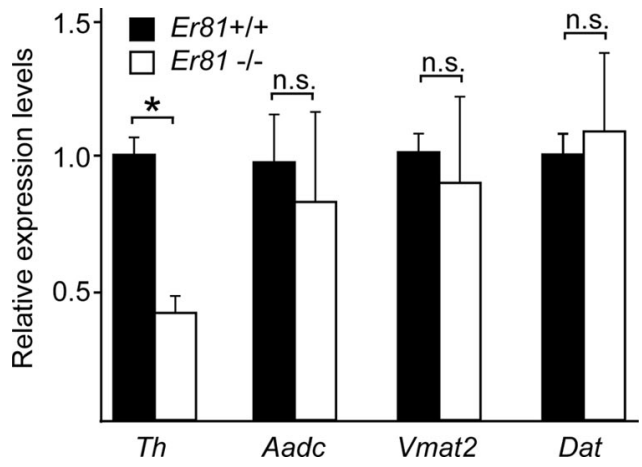

Figure 3. Expression of the dopaminergic gene cassette members in the $0 B$ of wild-type and Er81 mutant P10 mice as measured by quantitative RT-PCR. For each gene, expression levels are shown relative to the wild-type $0 \mathrm{~B}$. Gtpch expression was too low for reliable measurement and was not included in the analysis. Only Th expression was significantly decreased $(p<0.01)$ in the $\mathrm{OB}$ of the Er81 mutant mouse (indicated by asterisk), whereas levels for Aadc, Vmat2, and Dat were not significantly changed (n.s.).

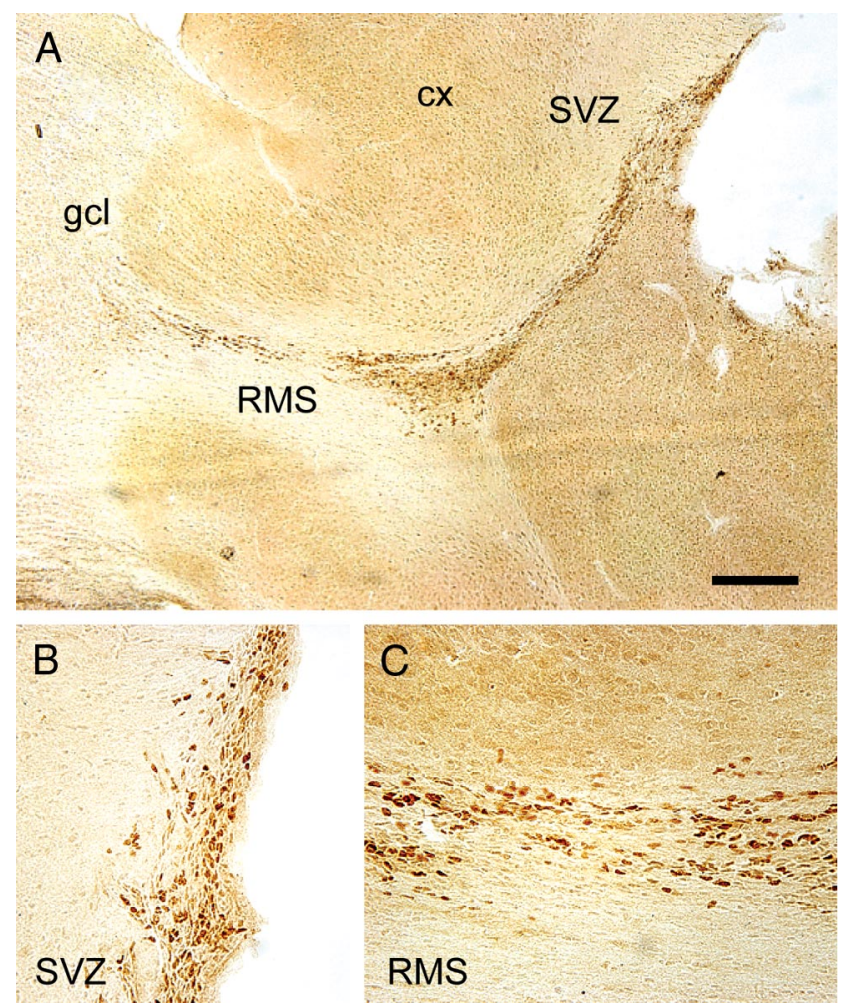

Figure 4. Immunohistochemical analysis of PEA3 in the forebrain. $A, P E A 3$ is expressed in the SVZ and RMS. B, C, Higher-magnification images of PEA3 immunoreactivity in the SVZ and RMS, respectively. PEA 3 is expressed in the nuclei of cells in SVZ and RMS, but no expression was observed in the OB granule cell layer (gCl) or cortex (cx). Scale bar: $A, 150 \mu \mathrm{m} ; \boldsymbol{B}, \boldsymbol{C}, 75 \mu \mathrm{m}$.

C57BL/6J mice subjected to unilateral naris occlusion or two sets of five postnatal day 10 (P10) mice that were either wild-type C57BL/6J or homozygous Er81 mutant.

For the nonquantitative analysis, reverse transcriptase (RT) and PCR amplification was performed using the SuperScript III One-Step kit (Invitrogen) following the protocol of the manufacturer. The following primers were used: $T h, 5^{\prime}$-CACTCCCTGTCAGAGGAGCC- $3^{\prime}$ and 5'-ATGAAGGGCAGGAGGAATGC-3'; Gtpch, 5' -TAAACTTGCCAGGATTGTAGAAATC- $3^{\prime}$ and $5^{\prime}$-CTTCAATCACTACTCCAACGCCA- ${ }^{\prime}$; Aadc, 5' -TTACATCCGAAAGCACGTGGAGCT-3' and 5' -AAGCAGACCAACCCAAGAATGACT-3'; Vmat2, 5'-TCATCGCTGCAGGCTCCATCT-3' and $5^{\prime}$-AGCTGCCACTTTCGGGAACAC-3'; and Dat, 


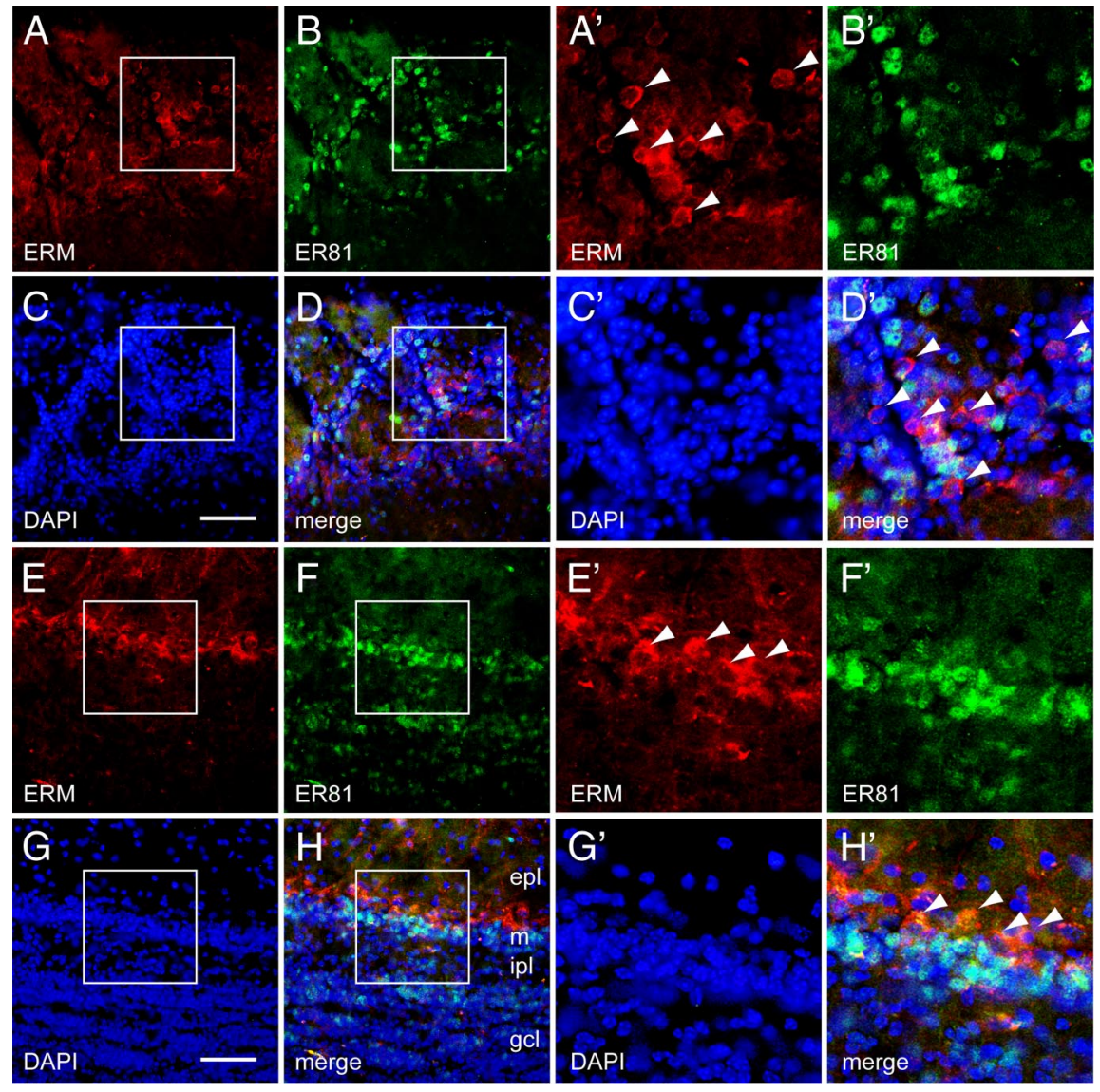

Figure 5. Immunofluorescence of ERM and ER81 expression in the OB. In either the glomerular layer $\left(\boldsymbol{A}-\boldsymbol{D}, \boldsymbol{A}^{\prime}-\boldsymbol{D}^{\prime}\right)$ or the mitral and granule cell layers $\left(\boldsymbol{E}-\boldsymbol{H}, \boldsymbol{E}^{\prime}-\boldsymbol{H}^{\prime}\right)$, cells containing ERM (arrowhead) did not coexpress ER81. These results suggest that ERM is not functionally redundant to ER81 in the $\mathrm{OB}$. epl, External plexiform layer; ipl, internal plexiform layer; gcl, granule cell layer; $\mathrm{m}$ mitral cell layer; DAPI, $\mathbf{4}^{\prime}, \mathbf{6}^{\prime}$-Diamidino-2-phenylindole. Scale bars: $\boldsymbol{A}-\boldsymbol{H}, 75 \mu \mathrm{m} ; \boldsymbol{A}^{\prime}-\boldsymbol{H}^{\prime}, 30 \mu \mathrm{m}$.

\section{5'-ACTTCAGGGAAGGTGGTGTGGAT-3' and 5'-GTAGAAGTCCA- CACTGAGGTATGC-3'.}

For quantitative analysis, first-strand reactions were conducted using SuperScript II first strand synthesis kit (Invitrogen), and the quantitative PCR reactions were performed on a 7500 Fast Real-Time PCR System (Applied Biosystems). Expression levels for Th, Er81, and glyceraldehyde3-phosphate dehydrogenase (Gapdh) were measured using Taqman Gene Expression Assay primer sets (Applied Biosystems) Mm00447557_m1, Mm00514804_m1, and Mm99999915_g1, respectively, with the TaqMan Universal PCR Master Mix (Applied Biosystems). For Aadc, Vmat2, and Dat, the primers described above were used, and their expression levels were measured with the SYBR Green PCR Master Mix (Applied Biosystems). Expression levels for all genes were normalized to Gapdh levels and reported as the mean, with error bars representing the SD. Data were analyzed using two-tailed Student's $t$ tests for each gene, and differences were considered significant if $p<0.01$.

Immunohistochemistry. Localization of single antigens was performed as described previously (Saino-Saito et al., 2007). Briefly, mice were anesthetized with an overdose of pentobarbital $(100 \mathrm{mg} / \mathrm{kg})$ and perfused transcardially with phosphate-buffered $4 \%$ formaldehyde, postfixed overnight, and then cryoprotected in $30 \%$ sucrose. Cryostat sections were fixed for 5 min with phosphate-buffered $4 \%$ formaldehyde and washed in PBS before being blocked with $1 \%$ bovine serum albumin in $\mathrm{PBS}$ and incubated overnight with primary antisera. Antigens were visualized by incubation with appropriate biotinylated secondary antiserum, the Vector Elite kit (Vector Laboratories), and 3,3'-diaminobenzidine (DAB) $(0.05 \%)$ as chromogen with hydrogen peroxide $(0.003 \%)$. Slides were dehydrated through a graded series of alcohols and coverslipped. The following primary antibodies and dilutions were used: rabbit anti-TH (lot 15-2, 1:25,000; raised in our laboratory); rabbit anti-ER81
[1:1000 for DAB immunohistochemistry (Covance); 1:1000 for immunofluorescence (kindly provided by Dr. Thomas Jessell)]; goat antiolfactory marker protein (OMP) (1:35,000; kindly provided by Frank Margolis, University of Maryland School of Medicine, Baltimore, MD); hamster anti-ERM (1:1000 for DAB immunohistochemistry or 1:500 for immunofluorescence; kindly provided by Kenneth Murphy, Washington University in St. Louis/ Howard Hughes Medical Institute, St. Louis, MO); rabbit anti-PEA3 (1:1000; Santa Cruz Biotechnology); rabbit anti-c-Fos (1:10,000; Calbiochem); and rabbit anti-PAX6 (1:1000; Covance).

Cell counts. TH-labeled cells in the OB were counted in equivalent sections from three wild-type and four Er81 mutant mice. PAX6labeled cells in the $\mathrm{OB}$ were counted within equivalent $1 \mathrm{~mm}$ glomerular regions from three wild-type and three Er81 mutant mice. Cell counts are expressed as the mean relative to the wild-type genotype, with error bars representing the SD. Data were analyzed by unpaired Student's $t$ test, and results were considered significantly different at $p<0.01$.

ChIP assays. For mice, two separate ChIP assays were performed using both bulbs in each assay. For dogs, each bulb from a single dog was separately used for two independent ChIP assays. OBs were dissected and fixed for $20 \mathrm{~min}$ in PBS and 1\% formaldehyde, after which the tissue was rinsed in PBS and then placed in lysis buffer (20 mm Tris, $\mathrm{pH} 8.1,150 \mathrm{~mm} \mathrm{NaCl}, 0.5 \%$ Triton $\mathrm{X}-100$, and $0.1 \%$ SDS). Tissue in the lysate suspension was crushed with a Dounce homogenizer before sonication with a Misonix 3000 sonicator. After sonication, cellular debris was removed via centrifugation, and lysate was then precleared with Protein A/G Sepharose beads (Santa Cruz Biotechnology). The lysate was then divided into two equivalent samples before adding $5 \mu \mathrm{g}$ of either anti-actin (Santa Cruz Biotechnology) or anti-ER81 (Covance). Antibody/lysate solutions were incubated with gentle rocking overnight at $4^{\circ} \mathrm{C}$, before Protein A/G Sepharose was added to precipitate antibody-protein-DNA complexes. The Protein A/G Sepharose beads were then removed from the lysate via centrifugation and washed twice with lysis buffer, twice with wash buffer (20 mu Tris, $\mathrm{pH} 8.1,150 \mathrm{~mm} \mathrm{NaCl}, 0.5 \%$ Triton X-100, 0.1\% SDS, and 2 mM EDTA), once with LiCl buffer $(0.25 \mathrm{M} \mathrm{LiCl}, 10 \mathrm{~mm}$ Tris, pH 8.1, $1 \mathrm{~mm}$ EDTA, 1\% NP-40, and 1\% deoxycholate), twice with TE (10 mM TrisEDTA, $\mathrm{pH} 8.0$, and $1 \mathrm{~mm}$ EDTA), and then placed in elution buffer $(0.1$ $\mathrm{M} \mathrm{NaHCO}_{3}, \mathrm{pH} 8.0,1 \%$ SDS, and $0.33 \mathrm{M} \mathrm{NaCl}$ ). The beads were incubated overnight in elution buffer at $65^{\circ} \mathrm{C}$. DNA was isolated from the elution buffer using Qiaquick PCR cleanup spin kits (Qiagen). To establish whether specific regions of genomic DNA containing known or predicted ER81 binding sites were immunoprecipitated, PCR reactions were performed using the following primers: mouse Th, 5'-TGGATGCAATTAGATCTAATGGGACGGAGG- $3^{\prime}$ and $5^{\prime}$-GCTCTGAGACGGCTCTTCTGAAGCCCTTGG-3'; mouse Mmp-13, 5'-CCCTCAGATTCTGCCACAAACCACAC-3' and 5'-GGATAGCTGAATGCATGGTGCCCAGC-3'; dog $T h, 5^{\prime}$-GTGATTCAGAGCGAGGGCCGCTG-3' and $5^{\prime}$-GAGGCGGTGTTGGGAGTGGGCAT-3'; and dog Mmp-13, 5'-TGTGCCTCCTTCACACACGTCCTG- ${ }^{\prime}$ and $5^{\prime}$-GCTTGCCTGTCCAGTGTCTCAGCG-3'.

EMSA. ER81 used in the EMSA experiments was generated by overexpression in recombinant pLysS Escherichia coli bacteria containing a pET-28A expression plasmid (Novagen) containing an Er81 cDNA isolated from mouse OB RNA using the Oligotex mRNA mini-kit (Qiagen). ER81 expression was induced with $1 \mathrm{~mm}$ isopropyl- $\beta$-D-thiogalac- 
topyranoside in bacterial cultures and harvested $6 \mathrm{~h}$ after induction. Cells were pelleted by centrifugation and then resuspended in 30 $\mathrm{ml}$ of Tris-buffered saline, $\mathrm{pH}$ 7.2. Resuspended cells were then sonicated, and cellular debris removed via centrifugation. Lysates were concentrated using centrifugal filtration to a final volume of $3 \mathrm{ml}$.

DNA oligos containing either the proximal or distal mouse consensus ER81 binding sites were synthesized so that there was a $4 \mathrm{bp} 5^{\prime}$ overhang containing one dT nucleotide. Oligos were labeled with ${ }^{32} \mathrm{P}$ via fill-in reaction of the $5^{\prime}$ overhang using $\left[\alpha-{ }^{2} \mathrm{P}\right] \mathrm{dATP}$ and Klenow fragment (New England Biolabs). Unincorporated nucleotides were removed using Bio-30 spin columns (Bio-Rad). The following oligo sequences were used: rodent (both rat and mouse) proximal site forward strand, $5^{\prime}$-GGAGAGGATGCGCAGGA-3'; rodent proximal site reverse strand, 5'-TCCTGCGCATCCTCTCCACGC-3'; human proximal site forward strand, 5'-GGGGTGGGGGATGTAAG-3'; and human proximal site reverse strand, $5^{\prime}$-CCTCCTTACATCCCCCACCCC- 3 '.

Recombinant protein lysate and labeled DNA oligos were incubated for $30 \mathrm{~min}$ at room temperature. Binding reactions consisted of 1 $\mu l$ of labeled DNA, $2 \mu$ of Herring sperm DNA $(10 \mathrm{mg} / \mathrm{ml}$ stock $), 2 \mu \mathrm{l}$ of BSA $(100 \mathrm{mg} / \mathrm{ml}$ stock), $1 \mu \mathrm{l}$ of NP-40, and 5, 10, 20, or $30 \mu \mathrm{l}$ of bacterial lysate. Binding reaction volumes were adjusted with Tris-buffered saline, $\mathrm{pH}$ 7.2, so that the final volume was $36 \mu \mathrm{l}$. Protein-DNA complexes were resolved on $4.5 \% 29: 1$ acrylamide/bis-acrylamide gels at a running voltage of 215 $\mathrm{V}$ for $2 \mathrm{~h}$. Gels were imaged using a BAS2500 phosphoimager (Fujifilm).

Mammalian Th promoter sequence analysis. Mammalian Th promoter nucleotide sequences were obtained from genomic assemblies NCBIm37, Rnor3.4, GRCh37, Btau4.0, and CanFam2.0 for the rat, mouse, human, cow, and dog, respectively, which are available from Ensembl (http://www.ensembl.org). Promoter regions were aligned using the MLAGAN alignment program available (http://lagan.stanford. edu) (Brudno et al., 2003).

\section{Results}

Basal expression levels of the dopaminergic gene cassette in the OB

Individual genes within the dopaminergic gene cassette are expressed at substantially different basal levels in the OB. Of the five genes in the cassette, RT-PCR analysis revealed that both Th and Dat were expressed at the highest levels, whereas Aadc and Vmat2 were expressed at substantially lower levels, and Gtpch was nearly undetectable (Fig. 1A). The extremely low Gtpch expression levels precluded additional analysis of this gene in the present study. The different expression levels of the individual members in the dopaminergic cassette are consistent with reported immunohistological analyses of these genes in the OB (Baker et al., 1991; Peter et al., 1995; Revay et al., 1996; Hwang et al., 1998). Despite these dramatically different expression levels, dopamine production in the OB was confirmed previously (Baker et al., 1983).

\section{Activity-dependent expression of the dopaminergic gene cassette in the $\mathrm{OB}$}

To test whether the expression levels of the dopamine gene cassette members were dependent on odor-mediated sensory activity, the $\mathrm{OB}$ of mice subjected to unilateral naris occlusion were analyzed by quantitative RT (qRT)-PCR. This analysis revealed that naris closure had no effect on the Aadc and Vmat2 expression, whereas Dat expression was slightly lowered and Th was strongly reduced (Fig. $1 B$ ). Also, ER81 protein levels were substantially downregulated in the $\mathrm{OB}$ ipsilateral to naris occlusion (Fig. 2), and qRT-PCR revealed a $72 \pm 22 \%$ decrease in Er81 mRNA expression levels. Thus, the non-uniform response in the expression of both Er81 and the gene cassette members to naris closure was inconsistent with the putative regulatory role of Er81 for these genes in the mouse OB.

\section{Regulation of dopaminergic gene cassette expression levels in Er81 mutant mice}

To determine whether Er81 was necessary for expression of the dopaminergic gene cassette members in the $\mathrm{OB}$, olfactory bulbs from homozygous Er81 mutant mice were analyzed by qRT-PCR. The mutant mouse strain used for this analysis contained an insertion in exon 11 of the Er81 gene that disrupts the ETS DNA binding domain and prevents the mutant protein from functioning as a transcription factor (Arber et al., 2000). Mice used for this analysis were $\sim 10 \mathrm{~d}$ old (P9-P11) rather than fetal or neonatal pups because the vast majority of $\mathrm{OB}$ interneurons are generated postnatally (Hinds, 1968). Examination of older mice was not possible because the homozygous Er81 mutant mice rarely lived past day P14.

The qRT-PCR analysis revealed that none of the genes in the dopaminergic cassette, except $T h$, were significantly downregulated in the $\mathrm{OB}$ of homozygous mutant mice relative to wild-type littermate controls (Fig. 3). These findings suggest that Er81 is only necessary for Th expression in the OB but not for the other members of the dopamine gene cassette.

\section{PEA3 and ERM expression in the $\mathrm{OB}$}

Er81 is a member of the PEA3 subfamily of ETS-domain transcription factors, which also contains Pea3 (Etv4) and Erm 

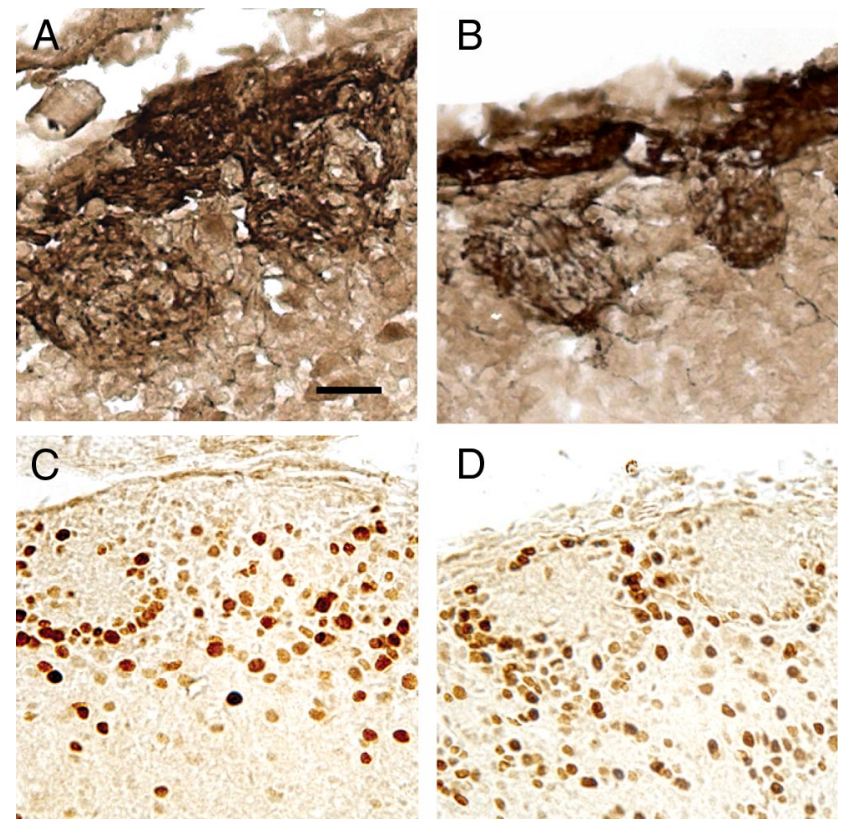

Figure 7. $0 \mathrm{MP}$ and c-Fos expression in the $\mathrm{OB}$ of Er81 mutant mice. $\boldsymbol{A}, \boldsymbol{B}, 0 \mathrm{MP}$ expression was detected in the glomeruli of both wild-type and Er81 mutant mice, respectively. This finding indicated that the loss of functional ER81 protein did not prevent innervation by the axons of olfactory receptor neurons. $\boldsymbol{C}, \boldsymbol{D}, \boldsymbol{C}$-Fos expression was detected in the glomerular layer of both wild-type and Er81 mice, respectively. Because c-Fos expression in the $\mathrm{OB}$ glomerular layer is activity dependent, this finding indicated that olfactory receptor neuron axons were functional and capable of transducing afferent synaptic activity. Scale bar, $40 \mu \mathrm{m}$.

\section{Regulation of Th expression by ER81 in the OB}

The results above clearly showed that Er81 was critical for proper expression of $T h$ in the OB. Immunohistochemical analysis revealed a $70 \pm 9 \%$ reduction in the number of TH-expressing cells in the $\mathrm{OB}$ of Er81 mutant mice $(240 \pm 43$ vs $72 \pm 21$ cells/OB section for $E r 81^{+/+}$and Er81 ${ }^{-/-}$, respectively) (Fig. $6 A, B$ ). However, because $\mathrm{TH}$ expression in the $\mathrm{OB}$ is activity dependent, the observed decrease in $\mathrm{TH}$ expression may have resulted from a disruption in afferent synaptic activity in the olfactory receptor neurons (ORNs). Immunostaining for OMP confirmed that ORN axons ramify within the glomeruli of the Er81 mutant mice (Fig. $7 A, B$ ). Furthermore, because c-Fos expression in the $\mathrm{OB}$ glomerular layer is activity dependent (Guthrie et al., 1993; Klintsova et al., 1995; Jin et al., 1996; Liu et al., 1999), the presence of c-Fos expression in the OB glomerular layer of mutant mice indicated that the loss of functional ER81 did not disrupt the synaptic activity of the ORNs (Fig. 7C,D).

Because ER81 is expressed in the neurogenic regions of the SVZ and RMS, the mutation of Er81 may have disrupted the generation and/or migration of $\mathrm{OB}$ dopaminergic neuronal progenitors and reduced $\mathrm{TH}$ expression in the OB. However, immunohistochemical analysis of PAX6 (Fig. 8A,B), which is coexpressed with $T h$ in OB neurons (Dellovade et al., 1998), revealed that PAX6-positive cell counts in the glomerular layer were unchanged in Er81 mutant mice $\left(86 \pm 12\right.$ cells $/ \mathrm{mm}^{2}$ for $\mathrm{Er} 81^{+/+}$vs $89 \pm 16$ cells $/ \mathrm{mm}^{2}$ for $\left.E r 81^{-/-}\right)$. Together these findings indicate that Er81 is critical for cell-autonomous regulation of Th expression in the OB dopaminergic neurons.

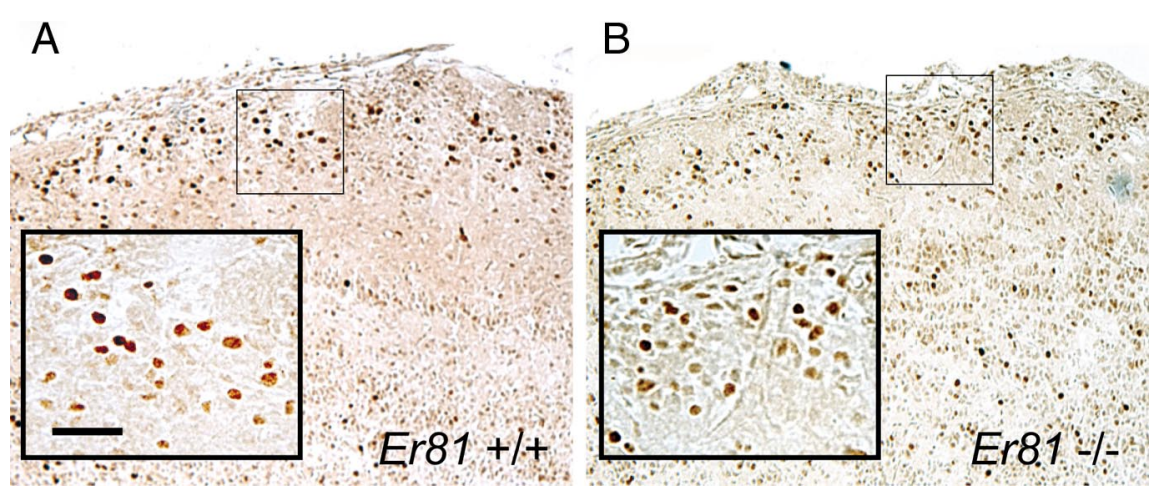

Figure 8. PAX6 expression in the $\mathrm{OB}$ of Er81 mutant mice. $\boldsymbol{A}, \boldsymbol{B}$, Immunohistochemistry revealed that there was no significant change in the number of PAX6-labeled cells in the glomerular layer of Er 81 mutant mice relative to wild-type littermates. Scale bar: $A, B, 125 \mu \mathrm{m}$; insets, $50 \mu \mathrm{m}$.

(Etv5). Because the amino acid sequences of the ETS DNA binding domain of all PEA3 group proteins are nearly identical, these proteins are likely to have identical binding sites (de Launoit et al., 1997). In the absence of functional ER81, either PEA3 or ERM might maintain expression of the dopamine gene cassette, except for $T h$, within the OB. However, immunohistological analysis revealed that PEA3 is not expressed in the OB but only in the SVZ and rostral migratory stream (RMS) (Fig. $4 A-C$ ). In contrast, immunofluorescence analysis found that ERM is expressed in the both the glomerular and mitral cell layers of the OB, but ERM is not coexpressed with ER81 in these layers (Fig. 5). Also, quantitative RT-PCR analysis of Erm and Pea3 gene expression in the $\mathrm{OB}$ of wild-type and Er81 mutant mice revealed the neither of these genes are upregulated in the mutant $\mathrm{OB}$ (data not shown).

\section{ER81 directly binds the rodent} Th promoter

Inspection of the $9 \mathrm{~kb}$ upstream regions of mouse and rat $T h$ genes identified two conserved consensus ER81 binding sites (Fig. 9A). Previous studies have shown that the $4.5 \mathrm{~kb}$ upstream region of the $T h$ gene is sufficient to mediate brain-regionspecific reporter gene expression in vivo (Schimmel et al., 1999), which suggested that only the consensus ER81 binding site in the proximal promoter is critical for OB-specific Th expression. The proximal consensus binding site also overlaps with the DA-motif (Fig. 9B) (Flames and Hobert, 2009). ChIP assays using mouse OB tissue indicated that the proximal consensus binding site is bound by ER81 in vivo (Fig. 9C). The previously identified and conserved ER81 binding site in the Mmp-13 gene (Mmp-1/collagenase) (supplemental Fig. S5, available at www.jneurosci.org as supplemental material) (Bosc et al., 2001) was used as a positive control (Fig. 9C). Because previous studies showed that the rodent proximal binding site is functional for mediating Th promoter activity in primary OB cells (Flames and Hobert, 2009), the ChIP findings in the current study suggest that ER81 regulates Th expression by directly binding the Th proximal promoter.

Although regulation of Th expression via ER81 binding to the DA-motif is suggested to be an evolutionarily conserved mechanism, analysis of the Th promoter from other mammals (humans, cows, and dogs) revealed that the proximal ER81 binding site/DA-motif is not conserved (Fig. 9B). The potential ER81 binding site in the human Th promoter conforms to the DA- 
A

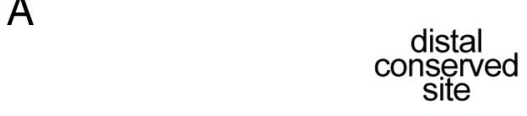

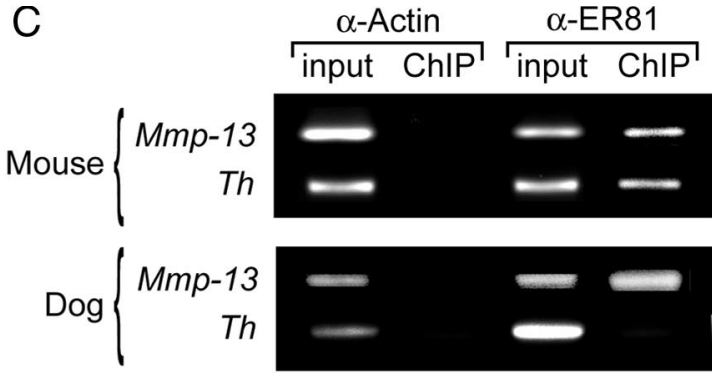

Human proximal site

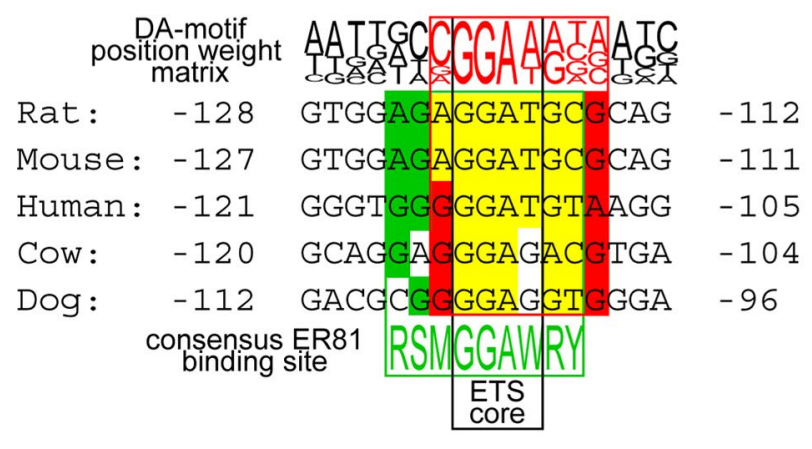

Rodent proximal site

D

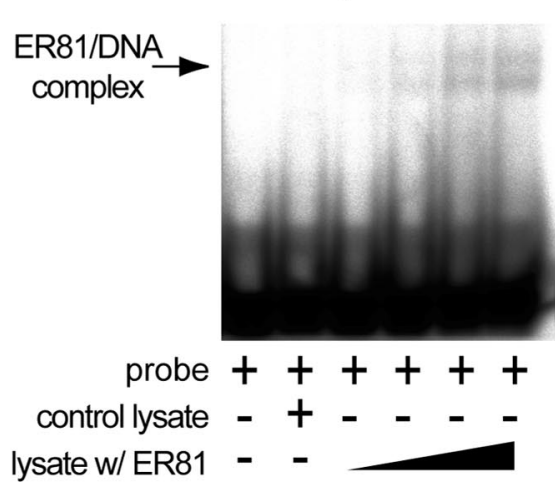

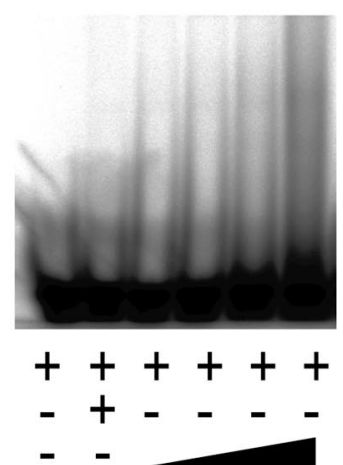

Figure 9. Species-specific ER81 binding to the Th proximal promoter. $\boldsymbol{A}$, Location of consensus ER81 binding sites in the $9 \mathrm{~kb}$ upstream regions of the rat and mouse Th genes. ER81 binding sites are indicated by " $E$," and conserved binding sites are indicated by shading between the two upstream regions. $\boldsymbol{B}$, Phylogenetic sequence comparison of equivalent $T h$ proximal promoter regions from rats, mice, humans, cows, and dogs. The ER81 consensus sequence (Brown and McKnight, 1992) is shaded in green, and the DA-motif position weight matrix (Flames and Hobert, 2009) is shaded in red. The core ETS DNA binding domain recognition sequence is boxed. Nucleotides that conform to both the DA-motif and ER81 consensus sequences are shaded yellow, whereas sequences that only conform to either the DA-motif or ER81 consensus sequence are shaded in red and green, respectively. Degenerate DNA code is as follows: $R=A / G ; S=C / G ; M=A / C ; W=A / T ; Y=C / T$. C, ChIP experiments with mouse OB tissue indicated that ER81 bound the mouse Th and Mmp-13 proximal promoters in vivo. In contrast, ChIP experiments with dog $0 \mathrm{~B}$ tissue indicated that ER81 bound only the Mmp-13, but not the Th, proximal promoters in vivo. The previously identified and conserved ER81 binding site in the Mmp-13 (Mmp-1/collagenase) proximal promoter was used as a positive control. D, EMSA revealed that bacterially expressed murine ER81 bound to the DA-motif/ER81 consensus binding site in the rodent proximal Th promoter but not the equivalent site in the human Th proximal promoter. The human and mouse ER81 protein amino acid sequences are 99\% identical, and the DNA binding domains of these proteins are $100 \%$ identical. Thus, the human and mouse ER81 proteins almost certainly have the same affinity for the binding sites in either the human or rodent Th promoters.

motif sequence, but the $5^{\prime}$ nucleotide adjacent to the core ETS recognition sequence is mutated and does not conform to the consensus ER81 binding site. Although the core ETS DNA binding sequence is conserved in both the rodent and human sequences, EMSAs revealed that ER81 was capable of only binding to the rodent, but not to the human, sequence (Fig. 9D). Compared with humans, the equivalent regions in the cow and $\operatorname{dog} T h$ promoters are even more divergent from the rodent sequence, including mutations to the core ETS biding motif(Fig. 9B). These observations suggest that it is unlikely that ER81 binds to either the cow or dog Th promoters.

ChIP assays were performed with dog OBs to test the whether ER81 binds the orthologous region in the dog Th proximal promoter. However, as shown in Figure 9C, ER81 binding detected with only the control binding site in the Mmp-13 and not with Th proximal promoter. Together, findings in this study suggested that the regulation of OB Th expression by the direct binding of ER81 to the Th promoter is a species-dependent molecular mechanism.

\section{Discussion}

\section{Regulation of the dopaminergic gene cassette by Er81}

This study showed that Er81 is necessary for the expression of the mouse $\mathrm{OB}$ dopaminergic neuron phenotype through the regulation of Th expression. In naris occluded mice, both mRNA and protein expression levels of Er81 and Th showed strong activity dependence. Th expression was also significantly reduced in Er81 mutant mice, and this reduction was not attributable to either a loss of afferent ORN activity or diminished proliferation/migration of progenitor cells. The reduction of Th was also not attributed to an increase in apoptosis because immunohistochemical analysis in the SVZ, RMS, and OB of wild-type and Er81 mutant littermates revealed no differences in the number of cells containing activated Caspase-3 (data not shown). Together, these findings suggest that Er81 regulates Th expression through a cellautonomous mechanism.

The ChIP and EMSA experiments in this study support a molecular mechanism in which ER81 regulates Th expression in rodents by directly binding to a conserved consensus binding site in the Th proximal promoter. However, this consensus binding site in the rodents is not conserved in the Th proximal promoters of other mammals (human, dog, and cow). In humans, the core ETS recognition sequence is present, but the $5^{\prime}$ nucleotide adjacent to the core motif is mutated and does not match the degenerate consensus binding site sequence. This mutation appears to render to the site non-functional since ER81 failed to bind the human sequence in EMSA experiments. The equivalent regions in the dog and cow Th promoter are even more divergent, and the ChIP assays with the dog OB tissue indicated that ER81 does not bind the dog Th promoter. Together, these findings suggest that ER81 does not regulate Th expression in these other mammals by direct binding to the proximal promoter. Thus, the molecular mechanism of ER81 directly binding the Th proximal promoter is likely species dependent. 
Although direct binding to the Th promoter may not be a conserved molecular mechanism, both the conservation of Er81 expression in the vertebrate brain (Chen et al., 1999; Zhu and Guthrie, 2002; Yoneshima et al., 2006; Langevin et al., 2007) and the coexpression of the Er81 ortholog ast-1 with Th in C. elegans (Flames and Hobert, 2009) suggest that regulation of the Th expression in the mammalian OB by Er81 is conserved. This conserved mechanism may involve ER81 binding to distal enhancer regions that are presently unidentified. Alternatively, ER81 may indirectly regulate $T h$ by activating genes that encode for transcription factors that directly bind to either enhancer or promoter regions of $T h$.

Unlike Th, expression of the other dopamine gene cassette members in the OB did not require Er81. Although Er81 expression levels were strongly activity dependent, Dat expression was only slightly activity dependent and Aadc and Vmat2 levels were insensitive to naris closure. The results for Aadc were consistent with previous in situ studies with unilateral naris occluded rats (Stone et al., 1990). Analysis of Er81 mutant mice showed that neither Aadc, Vmat2, nor Dat expression levels were significantly decreased by the loss of functional ER81. In contrast to a recently proposed model for differentiation of dopaminergic neurons (Flames and Hobert, 2009), these findings indicate that Er81 is not necessary for the expression of Aadc, Vmat2, or Dat in the mouse OB.

\section{Role of the DA-motif in differentiation of dopaminergic $\mathrm{OB}$ interneurons}

ER81 has been suggested to regulate expression of the dopaminergic cassette genes in the OB by direct binding to dopaminemotif nucleotide sequences that are near the transcription start site in these genes (Flames and Hobert, 2009). Although the DAmotif sequence overlaps with the established ER81 consensus binding site, our study does not support a functional role for Er81 in the regulation of Aadc, Vmat2, or Dat. Consistent with this finding, ChIP assays with mouse OB tissue suggest that ER81 does not bind identified DA-motifs in Vmat2, Dat, and Gtpch (supplemental Fig. S6, available at www.jneurosci.org as supplemental material). Furthermore, the DA-motifs identified near the mouse Aadc, Vmat2, Dat, and Gtpch genes are not conserved in other mammals (supplemental Figs. S1-S4, available at www.jneurosci. org as supplemental material), and ChIP assays with dog OB tissue do not immunoprecipitate the regions of dog dopaminergic cassette genes that are orthologous to the mouse genes that contain DA-motifs (supplemental Fig. S6, available at www. jneurosci.org as supplemental material). These results, together with the findings that ER81 is not necessary for expression of the entire dopaminergic gene cassette in the OB, do not support a significant role for the DA-motif in the differentiation of mammalian $\mathrm{OB}$ dopaminergic interneurons.

There is little or no evidence suggesting a role for the DAmotif in differentiation of dopaminergic neurons outside of the OB. The functionality of the DA-motif/ER81 consensus binding sites have not been demonstrated in dopaminergic neurons from other regions, such as the substantia nigra. The lack of conservation in the various mammalian dopaminergic gene cassette promoters also suggests that the DA-motif may not be significant for dopamine neurons in general. The ER81 homolog Erm (Etv5) has been suggested to mediate dopaminergic differentiation in mammalian non-OB dopaminergic neurons (Flames and Hobert, 2009), but there is no reported evidence that ERM is coexpressed with, or recruited to the regulatory regions of, the dopaminergic cassette genes.

\section{Implications for molecular transcription codes for $\mathrm{OB}$ interneuron phenotypes}

In the OB glomerular layer, ER81 is coexpressed with both calretinin and $\mathrm{TH}$, but there is no coexpression of calretinin and $\mathrm{TH}$ (Kosaka et al., 1995; Parrish-Aungst et al., 2007). These observations indicate that ER81 is necessary but not sufficient for the OB dopaminergic phenotype. Previous studies have proposed that combinatorial molecular codes of transcription factors specify various glomerular layer interneuron phenotypes (Allen et al., 2007). These studies have suggested that a combination of ER81, PAX6, and MEIS2 expression forms a molecular code for OB dopaminergic interneurons because nearly all cells containing $\mathrm{TH}$ also express each of these factors. However, this current study revealed that the loss of ER81 did not completely eliminate $\mathrm{TH}$ expression in the $\mathrm{OB}$ ( $\mathrm{TH}$-immunoreactive cell counts were $\sim 30 \%$ of wild-type controls). This observation suggests that there are other transcription factors that are redundant to ER81 in a subset of TH-expressing cells. However, paralogs of ER81, PEA3, and ERM were not expressed in the proper OB cell types and thus do not account for this redundancy. Thus, there may be either other ETS DNA binding domain proteins or an entirely different class of transcription factors that are capable of replacing ER81 in a subset of OB dopaminergic neurons. An alternative, but not mutually exclusive, possibility is that the remaining $\mathrm{TH}$ expressing cells in the $\mathrm{OB}$ of Er81 mutant may represent a subpopulation of $\mathrm{OB}$ dopaminergic cells that have derived from an alternative origin. Recent studies have suggested that regions other than the LGE generate OB dopaminergic interneurons during embryonic development (Vergaño-Vera et al., 2006; Inoue et al., 2007). The progenitors derived from these alternative origins may not depend on ER81 for Th expression.

This complexity in the molecular-genetic pathways that regulate $\mathrm{OB}$ dopaminergic interneuron differentiation have also been reported for other OB interneuron phenotypes. Nearly all calretinin-expressing interneurons express SP8, but calretinin expression is lost from only approximately one-half of these cells in mice lacking SP8 (Waclaw et al., 2006). Together, these findings suggest that there may be multiple molecular transcription codes for individual glomerular interneuron phenotypes. Identification of the molecular transcription codes and elucidation of the mechanisms by which they regulate specific interneuron phenotypes remains a major challenge.

\section{References}

Allen ZJ 2nd, Waclaw RR, Colbert MC, Campbell K (2007) Molecular identity of olfactory bulb interneurons: transcriptional codes of periglomerular neuron subtypes. J Mol Histol 38:517-525.

Anderson S, Mione M, Yun K, Rubenstein JL (1999) Differential origins of neocortical projection and local circuit neurons: role of Dlx genes in neocortical interneuronogenesis. Cereb Cortex 9:646-654.

Arber S, Ladle DR, Lin JH, Frank E, Jessell TM (2000) ETS gene Er81 controls the formation of functional connections between group Ia sensory afferents and motor neurons. Cell 101:485-498.

Baker H, Kawano T, Margolis FL, Joh TH (1983) Transneuronal regulation of tyrosine hydroxylase expression in olfactory bulb of mouse and rat. J Neurosci 3:69-78.

Baker H, Abate C, Szabo A, Joh TH (1991) Species-specific distribution of aromatic L-amino acid decarboxylase in the rodent adrenal gland, cerebellum, and olfactory bulb. J Comp Neurol 305:119-129.

Baker H, Morel K, Stone DM, Maruniak JA (1993) Adult naris closure profoundly reduces tyrosine hydroxylase expression in mouse olfactory bulb. Brain Res 614:109-116.

Bosc DG, Goueli BS, Janknecht R (2001) HER2/Neu-mediated activation of the ETS transcription factor ER81 and its target gene MMP-1. Oncogene 20:6215-6224.

Brown TA, McKnight SL (1992) Specificities of protein-protein and 
protein-DNA interaction of GABP alpha and two newly defined etsrelated proteins. Genes Dev 6:2502-2512.

Brudno M, Do CB, Cooper GM, Kim MF, Davydov E, Green ED, Sidow A, Batzoglou S (2003) LAGAN and Multi-LAGAN: efficient tools for largescale multiple alignment of genomic DNA. Genome Res 13:721-731.

Cave JW, Baker H (2008) Dopamine systems in the forebrain. In: Development and engineering of dopamine neurons (Pasterkamp RJ, Smidt MP, Burbach JPH, eds). Austin, TX: Landes BioScience.

Chen Y, Hollemann T, Grunz H, Pieler T (1999) Characterization of the Ets-type protein ER81 in Xenopus embryos. Mech Dev 80:67-76.

de Launoit Y, Baert JL, Chotteau A, Monte D, Defossez PA, Coutte L, Pelczar H, Leenders F (1997) Structure-function relationships of the PEA3 group of Ets-related transcription factors. Biochem Mol Med 61:127-135.

Dellovade TL, Pfaff DW, Schwanzel-Fukuda M (1998) Olfactory bulb development is altered in small-eye (sey) mice. J Comp Neurol 402: 402-418.

Flames N, Hobert O (2009) Gene regulatory logic of dopamine neuron differentiation. Nature 458:885-889.

Guthrie KM, Anderson AJ, Leon M, Gall C (1993) Odor-induced increases in c-fos mRNA expression reveal an anatomical "unit" for odor processing in olfactory bulb. Proc Natl Acad Sci U S A 90:3329-3333.

Hinds JW (1968) Autoradiographic study of histogenesis in the mouse olfactory bulb. I. Time of origin of neurons and neuroglia. J Comp Neurol 134:287-304.

Hwang O, Baker H, Gross S, Joh TH (1998) Localization of GTP cyclohydrolase in monoaminergic but not nitric oxide-producing cells. Synapse 28:140-153.

Inoue T, Ota M, Ogawa M, Mikoshiba K, Aruga J (2007) Zic1 and Zic3 regulate medial forebrain development through expansion of neuronal progenitors. J Neurosci 27:5461-5473.

Jin BK, Franzen L, Baker H (1996) Regulation of c-Fos mRNA and fos protein expression in olfactory bulbs from unilaterally odor-deprived adult mice. Int J Dev Neurosci 14:971-982.

Klintsova AY, Philpot BD, Brunjes PC (1995) Fos protein immunoreactivity in the developing olfactory bulbs of normal and naris-occluded rats. Brain Res Dev Brain Res 86:114-122.

Kosaka K, Aika Y, Toida K, Heizmann CW, Hunziker W, Jacobowitz DM, Nagatsu I, Streit P, Visser TJ, Kosaka T (1995) Chemically defined neuron groups and their subpopulations in the glomerular layer of the rat main olfactory bulb. Neurosci Res 23:73-88.

Langevin LM, Mattar P, Scardigli R, Roussigné M, Logan C, Blader P, Schuurmans C (2007) Validating in utero electroporation for the rapid analysis of gene regulatory elements in the murine telencephalon. Dev Dyn 236:1273-1286.

Liu N, Cigola E, Tinti C, Jin BK, Conti B, Volpe BT, Baker H (1999) Unique regulation of immediate early gene and tyrosine hydroxylase expression in the odor-deprived mouse olfactory bulb. J Biol Chem 274:3042-3047.

Lois C, Alvarez-Buylla A (1994) Long-distance neuronal migration in the adult mammalian brain. Science 264:1145-1148.
Luskin MB (1993) Restricted proliferation and migration of postnatally generated neurons derived from the forebrain subventricular zone. Neuron 11:173-189.

Parrish-Aungst S, Shipley MT, Erdelyi F, Szabo G, Puche AC (2007) Quantitative analysis of neuronal diversity in the mouse olfactory bulb. J Comp Neurol 501:825-836.

Peter D, Liu Y, Sternini C, de Giorgio R, Brecha N, Edwards RH (1995) Differential expression of two vesicular monoamine transporters. J Neurosci 15:6179-6188.

Revay R, Vaughan R, Grant S, Kuhar MJ (1996) Dopamine transporter immunohistochemistry in median eminence, amygdala, and other areas of the rat brain. Synapse 22:93-99.

Saino-Saito S, Cave JW, Akiba Y, Sasaki H, Goto K, Kobayashi K, Berlin R, Baker H (2007) ER81 and CaMKIV identify anatomically and phenotypically defined subsets of mouse olfactory bulb interneurons. J Comp Neurol 502:485-496.

Schimmel JJ, Crews L, Roffler-Tarlov S, Chikaraishi DM (1999) $4.5 \mathrm{~kb}$ of the rat tyrosine hydroxylase $5^{\prime}$ flanking sequence directs tissue specific expression during development and contains consensus sites for multiple transcription factors. Brain Res Mol Brain Res 74:1-14.

Stenman J, Toresson H, Campbell K (2003) Identification of two distinct progenitor populations in the lateral ganglionic eminence: implications for striatal and olfactory bulb neurogenesis. J Neurosci 23:167-174.

Stone DM, Wessel T, Joh TH, Baker H (1990) Decrease in tyrosine hydroxylase, but not aromatic L-amino acid decarboxylase, messenger RNA in rat olfactory bulb following neonatal, unilateral odor deprivation. Brain Res Mol Brain Res 8:291-300.

Vergaño-Vera E, Yusta-Boyo MJ, de Castro F, Bernad A, de Pablo F, VicarioAbejón C (2006) Generation of GABAergic and dopaminergic interneurons from endogenous embryonic olfactory bulb precursor cells. Development 133:4367-4379.

Waclaw RR, Allen ZJ 2nd, Bell SM, Erdélyi F, Szabó G, Potter SS, Campbell K (2006) The zinc finger transcription factor Sp8 regulates the generation and diversity of olfactory bulb interneurons. Neuron 49:503-516.

Weihe E, Depboylu C, Schütz B, Schäfer MK, Eiden LE (2006) Three types of tyrosine hydroxylase-positive CNS neurons distinguished by dopa decarboxylase and VMAT2 co-expression. Cell Mol Neurobiol 26:659-678.

Wichterle H, Turnbull DH, Nery S, Fishell G, Alvarez-Buylla A (2001) In utero fate mapping reveals distinct migratory pathways and fates of neurons born in the mammalian basal forebrain. Development 128:37593771.

Yoneshima H, Yamasaki S, Voelker CC, Molnár Z, Christophe E, Audinat E, Takemoto M, Nishiwaki M, Tsuji S, Fujita I, Yamamoto N (2006) Er81 is expressed in a subpopulation of layer 5 neurons in rodent and primate neocortices. Neuroscience 137:401-412.

Zhu Y, Guthrie S (2002) Expression of the ETS transcription factor ER81 in the developing chick and mouse hindbrain. Dev Dyn 225:365-368. 